Original Paper http://ajol.info/index.php/ijbcs http://indexmedicus.afro.who.int

\title{
Evaluation nutritionnelle de quelques ingrédients entrant dans la formulation alimentaire des poules pondeuses et porcs d'une ferme d'élevage au Nord- Ouest Cameroun
}

\author{
Roger PONKA ${ }^{1 *}$, Augustin GOUDOUM ${ }^{1}$, Aristide CHAMI TCHUNGOUELIEU ${ }^{1}$ et \\ Elie $\mathrm{FOKOU}^{2}$
}
${ }^{1}$ Département Agriculture Elevage et des Produits Dérivés, Institut Supérieur du Sahel, Université de Maroua BP 46, Maroua, Cameroun.
${ }^{2}$ Département de Biochimie, Faculté des Sciences, Université de Yaoundé I, BP 812 Yaoundé, Cameroun. *Auteur correspondant ; E-mail : rponka@yahoo.fr; Tel: 00237677974324

\section{RESUME}

Les provendes constituent la principale composante de dépense en aviculture et en élevage porcin. On constate sur le marché une différence de prix pour un même ingrédient donné, livré par des fournisseurs. Ainsi, dans l'optique d'orienter les fermiers pour le choix des ingrédients de hautes valeurs nutritionnelles et de moindre coût, le présent travail a pour objectif d'établir une base de données des valeurs nutritionnelles de quelques ingrédients (tourteaux de soja, farine de poisson, balles de riz, tourteau d'arachide et aliment ponte) entrant dans la formulation alimentaire des poules pondeuses et porcs. Ainsi, les différents échantillons ont été collectés auprès des fournisseurs et les aliquotes ont été prélevés pour les analyses au laboratoire. Les teneurs en eau, cendres, protéines et lipides ont été déterminées selon les méthodes AOAC. La teneur en glucide a été déterminée par différence. A l'issue de ces analyses, il ressort que les teneurs exprimées en $\mathrm{g} / 100 \mathrm{~g}$ se trouvent dans les fourchettes suivantes : eau 8,86-16,65; cendres 3,93-18,98; protéines 5,51-63,73; lipides 8,53-26,96; glucide, 0,0-61,18 et énergie 341,23-465,54 kcal/100g. Ces données pourraient orienter les fermiers pour le choix des ingrédients de hautes valeurs nutritionnelles et de moindre coût.

(C) 2016 International Formulae Group. All rights reserved.

Mots clés : Valeur nutritionnelle, ingrédients, poules pondeuse, porcs.

\section{Nutritional Evaluation of some ingredients using in food the formulation of laying hens and pigs from the breeding farm in the North-West Cameroon}

\begin{abstract}
Animal feed is the main component of expenditure in poultry and pig breeding. Also, there is a difference in the market price for a given ingredient supplied by the same supplier. Thus, in the aim of guiding farmers to the choice of ingredients with high nutritional value and lower cost, the present work aims to establish a database of nutritional values of some ingredients (soybean meal, fish meal, rice husks, peanut meal and food egg) used in the formulation of food laying hens and pigs. Thus, the different samples were collected from suppliers and aliquots were taken for laboratory analysis. The contents of moisture, ash, protein and fat
\end{abstract}


were determined according to AOAC methods. The carbohydrate content was determined by difference. Following these analyzes, it appears that the contents expressed in $\mathrm{g} / 100 \mathrm{~g}$ were in the following ranges: moisture, 8.86 g-16.65; ash 3.93-18.98 ; protein, 5.51-63.73 ; fat, 8.53-26.96 ; carbohydrate, 0.0-61.18 and energy, 341.23-465.54 kcal/100g. These data could guide farmers for the choice of ingredients of high nutritional value and low cost.

(C) 2016 International Formulae Group. All rights reserved.

Keyswords: Nutritional value, ingredients, laying hens, pigs.

\section{INTRODUCTION}

Jusqu'au début de ce troisième millénaire, la malnutrition ne cesse de s'accentuer dans les pays en voie de développement, malgré l'évolution des sciences dans le domaine de l'agroalimentaire (FAO, 2004). Dans sa campagne mondiale de lutte contre la faim, la FAO a montré qu'elle a progressée dans le monde en raison de la crise économique mondiale de 2008-2009 et touche environ 1,2 milliards de personnes, soit un sixième de la population mondiale, dont les 307 millions sont des africains (Jeune Afrique, 2010). En outre, le nombre de personnes sous alimentées est en nette croissance en Afrique et si rien n'est fait dans la vision d'atteindre les objectifs du millénaire pour le développement, plusieurs millions d'africains continueront à souffrir du déficit nutritionnel.

Par ailleurs, la FAO avait montré que les carences en protéines plus particulièrement d'origine animale figurent parmi les carences nutritionnelles les plus répandues dans le monde (FAO, 2004). Ainsi, selon la FAO et l'OMS, la quantité de protéines animales nécessaires pour chaque individu est de 42 $\mathrm{kg} / \mathrm{hab} / \mathrm{an}$. Or, au Cameroun, le secteur viande n'apporte que $34 \mathrm{~kg} / \mathrm{hab} / \mathrm{an}$ avec une consommation moyenne de $18 \mathrm{~kg} / \mathrm{hab} / \mathrm{an}$ (SSEPIA/MINEPIA, 2002 ; FAO, 2005). D'où l'urgence et la nécessité pour le gouvernement camerounais de promouvoir la production animale et plus particulièrement l'élevage à cycle court (aviculture, élevage porcin etc.), afin de satisfaire les besoins nutritionnels des populations et dégager des excédents pour l'exportation (DSCE, 2010).

L'aviculture et l'élevage porcin nécessitent un certain nombre d'exigences qui ont un coût à savoir: le bâtiment, l'équipement (abreuvoir, mangeoire), les animaux, le suivi sanitaire et l'alimentation. Cependant, l'alimentation constitue la principale composante de dépense en aviculture et en élevage porcin car, elle représente 70 à $80 \%$ des coûts de production dans ces élevages et joue un rôle prépondérant sur les performances et les qualités des produits (Sonaiya et Swan, 2004; Ndébi et al., 2009).

L'effort pour formuler et produire un aliment de qualité passe nécessairement par une bonne connaissance de la composition des matières premières et des causes de leur variabilité en valeur nutritive. Contrairement aux pays occidentaux qui disposent des tables publiques de composition des matières premières alimentées par une masse considérable de résultats d'analyses (Ngom, 2004), le Cameroun ne dispose à l'heure actuelle que très peu de données similaires. Ainsi, dans l'optique de proposer aux fermiers, les ingrédients de hautes valeurs nutritionnelles au moindre coût, le présent travail a pour objectif d'établir une base de données des valeurs nutritionnelles de quelques ingrédients (tourteaux de soja, farine de poisson, balles de riz, tourteau d'arachide et aliment ponte) entrant dans la formulation alimentaire des poules pondeuses et porcs. 


\section{MATERIEL ET METHODES Zone d'étude}

Bamenda est situé entre les $9^{\circ} 13$ et $11^{\circ} 26$ de la longitude Est et les $4^{\circ} 53$ et $7^{\circ} 53$ de la latitude Nord. Le climat qui y règne est $\mathrm{du}$ type tropical humide. Les hauteurs annuelles de pluies sont en moyennes de 2000 $\mathrm{mm}$; les températures varient entre de $15^{\circ} \mathrm{C}$ à $25{ }^{\circ} \mathrm{C}$ avec une humidité relative allant de 53 à $97 \%$. La ville de Bamenda est sous l'influence de deux saisons: une longue saison de pluies (mi-mars à mi- novembre) et une courte saison sèche de mi-novembre à mimars (Donfack et al., 1996).

\section{Matériel biologique}

Le matériel biologique était constitué des échantillons suivants: les tourteaux de soja $\mathrm{A}$, B et $\mathrm{C}$ provenant de trois fournisseurs dénommés ( $\mathrm{A}, \mathrm{B}$ et $\mathrm{C}$ ); des farines de poissons $\mathrm{D}$ et $\mathrm{E}$ de deux fournisseurs dénommés ( $D$ et $E$ ); des balles de riz; de tourteaux d'arachides et de l'aliment ponte destiné aux poules pondeuses en période de ponte. Tous ces aliments ont été prélevés auprès des fournisseurs d'ingrédients de Bamenda.

\section{Echantillonnage}

La collecte des échantillons a été faite auprès des fournisseurs livrant les ingrédients aux fermiers et au niveau de l'unité de transformation des aliments de la ferme. Les échantillons ont été conditionnés dans les emballages plastiques en polyéthylène de couleur sombre pour empêcher l'absorption d'eau et l'oxydation des lipides en présence de la lumière. Par la suite, ils ont été transportés au Laboratoire d'analyse du Centre de Recherche en Alimentation de Nutrition de l'Institut de recherche Médicale et d'étude des Plantes Médicinales à Yaoundé pour analyses chimiques.
Teneurs en eau, cendres, protéines, lipides, et glucides

Les teneurs en eau, cendres, protéines et lipides ont été déterminées selon les méthodes AOAC (1980). La teneur en eau a été déterminée par séchage des échantillons à l'étuve à $105{ }^{\circ} \mathrm{C}$ jusqu'à obtention d'un poids constant. La teneur en cendres, par incinération au four à $550{ }^{\circ} \mathrm{C}$ pendant $48 \mathrm{~h}$. En ce qui concerne la teneur en lipides, elle a été déterminée par extraction au soxhlet avec de l'éther pétrole $\left(40-60{ }^{\circ} \mathrm{C}\right)$ pendant $6 \mathrm{~h}$. La teneur en protéines a été déterminée par la méthode de Kjeldahl. Toutes les analyses ont été effectuées en triple. La teneur en glucides a été calculée par différence.

\section{Analyse statistique des résultats}

Les résultats des analyses sont présentés sous forme de moyenne \pm écarttype. Le traitement des résultats s'est fait par analyse des variances (ANOVA) au seuil de significativité $P<0.05$. Ces tests ont été effectués à l'aide du logiciel SPSS for Windows version 17.0.

\section{RESULTATS}

Les résultats des analyses sont présentés dans les Tableaux 1 et 2 . Le Tableau 1 donne les teneurs en eau, matière sèche, cendres et en matière organique exprimées en $\mathrm{g} / 100 \mathrm{~g}$ d'aliment des différents échantillons. Le Tableau 2 quant à lui présente les teneurs en lipides, protéines et glucides exprimées en $\mathrm{g} / 100 \mathrm{~g}$ d'aliment et les valeurs énergétiques exprimées en $\mathrm{kcal} / 100 \mathrm{~g}$ d'aliment. Les teneurs en eau des aliments varient de 8,86 (farine de poisson E) à $16,65 \mathrm{~g} / 100 \mathrm{~g}$ (tourteau de soja C). Toutefois, les teneurs en eau du tourteau de soja A, tourteau de soja B, farine de poisson $\mathrm{D}$, tourteau d'arachide, balles de riz, aliment ponte sont respectivement de 11,41; 12,$05 ; 12,24 ; 9,84 ; 12,05 ; 11,34$ g/100g. Les 
teneurs en cendres des ingrédients varient de 3,93 (tourteau de soja A) à 18,98 g/100g (farine de poisson E). Le tourteau de soja B a une teneur en cendres de $5,25 \%$; tourteau de soja C, $5,02 \%$; farine de poisson D, $16,46 \%$; tourteau d'arachide, 4,38\%; balles de riz, $9,57 \%$ et aliment ponte $6,96 \mathrm{~g} / 100 \mathrm{~g}$. Les teneurs en lipides varient de 8,53 (tourteau de soja A) à 26,96 g/100g (tourteau de soja B). La teneur en protéines la plus faible $(5,51$ $\mathrm{g} / 100 \mathrm{~g}$ ) se retrouve dans les balles de riz et la plus élevée $(63,73 \mathrm{~g} / 100 \mathrm{~g})$ dans la farine de poisson D. En ce qui concerne les glucides, la teneur la plus élevée $(61,18 \mathrm{~g} / 100 \mathrm{~g})$ a été obtenue dans les balles de riz alors que la plus faible $(00,00 \mathrm{~g} / 100 \mathrm{~g})$ est celle de la farine de poisson D. La valeur énergétique des aliments varient de $341,23 \mathrm{kcal} / 100 \mathrm{~g}$ pour la farine de poisson $\mathrm{D}$ à $465,54 \mathrm{kcal} / 100 \mathrm{~g}$ pour le tourteau de soja B. En ce qui concerne, les tourteaux de soja, les valeurs énergétiques oscillent entre 381,25 et $435,05 \mathrm{kcal} / 100 \mathrm{~g}$.

Tableau 1: Teneurs en eau, matière sèche, cendres, matière organique des échantillons $(\mathrm{g} / 100 \mathrm{~g})$.

\begin{tabular}{lllll}
\hline Echantillon & Teneur en eau & $\begin{array}{l}\text { Teneur en } \\
\text { matière sèche }\end{array}$ & $\begin{array}{l}\text { Teneur en } \\
\text { cendres }\end{array}$ & $\begin{array}{l}\text { Teneur en matière } \\
\text { organique }\end{array}$ \\
\hline Tourteau de Soja A & $11,41 \pm 0,21^{\mathrm{c}}$ & $88,59 \pm 0,21^{\mathrm{c}}$ & $3,93 \pm 0,18^{\mathrm{g}}$ & $84,66 \pm 0,06^{\mathrm{f}}$ \\
Tourteau de Soja B & $12,07 \pm 0,21^{\mathrm{b}}$ & $87,93 \pm 0,21^{\mathrm{d}}$ & $5,25 \pm 0,04^{\mathrm{e}}$ & $82,68 \pm 0,19^{\mathrm{a}}$ \\
Tourteau de Soja C & $16,65 \pm 0,14^{\mathrm{a}}$ & $83,35 \pm 0,14^{\mathrm{e}}$ & $5,02 \pm 0,04^{\mathrm{ef}}$ & $78,33 \pm 0,18^{\mathrm{b}}$ \\
Farine de Poisson D & $12,24 \pm 0,20^{\mathrm{b}}$ & $87,76 \pm 0,20^{\mathrm{d}}$ & $16,46 \pm 0,53^{\mathrm{b}}$ & $71,30 \pm 0,39^{\mathrm{e}}$ \\
Farine Poisson E & $8,86 \pm 0,12^{\mathrm{e}}$ & $91,14 \pm 0,12^{\mathrm{a}}$ & $18,98 \pm 0,67^{\mathrm{a}}$ & $72,16 \pm 0,56^{\mathrm{d}}$ \\
Tourteau d'Arachide & $9,84 \pm 0,04^{\mathrm{d}}$ & $90,16 \pm 0,04^{\mathrm{b}}$ & $4,38 \pm 0,16^{\mathrm{fg}}$ & $85,78 \pm 0,19^{\mathrm{c}}$ \\
Balles de Riz & $12,05 \pm 0,04^{\mathrm{b}}$ & $87,95 \pm 0,04^{\mathrm{d}}$ & $9,57 \pm 0,39^{\mathrm{c}}$ & $78,39 \pm 0,35^{\mathrm{d}}$ \\
Aliment Ponte & $11,34 \pm 0,00^{\mathrm{c}}$ & $88,66 \pm 0,00^{\mathrm{c}}$ & $6,96 \pm 0,04^{\mathrm{d}}$ & $81,69 \pm 0,04^{\mathrm{d}}$ \\
\hline
\end{tabular}

Les moyennes situées dans la même colonne et portant les lettres différentes en exposant sont significativement différentes au seuil $P<0,05$.

Tableau 2 : Teneur en lipides, protéines, glucides des échantillons $(\mathrm{g} / 100 \mathrm{~g})$ et valeur énergétique en $\mathrm{kcal} / 100 \mathrm{~g}$.

\begin{tabular}{lcccc}
\hline Echantillon & Lipides & Protéines & Glucides & Valeur Energétique \\
\hline Tourteau de soja A & $8,53 \pm 0,19^{\mathrm{f}}$ & $39,38 \pm 0,33^{\mathrm{c}}$ & 36,75 & 381,26 \\
Tourteau de soja B & $26,96 \pm 0,14^{\mathrm{a}}$ & $39,15 \pm 0,68^{\mathrm{c}}$ & 16,56 & 465,54 \\
Tourteau de soja C & $24,35 \pm 0,21^{\mathrm{b}}$ & $36,12 \pm 0,60^{\mathrm{d}}$ & 17,86 & 435,05 \\
Farine de poisson D & $9,59 \pm 0,21^{\mathrm{e}}$ & $63,73 \pm 0,80^{\mathrm{a}}$ & 00,00 & 341,23 \\
Farine de poisson E & $11,99 \pm 0,07^{\mathrm{d}}$ & $55,69 \pm 1,65^{\mathrm{b}}$ & 4,48 & 348,58 \\
Tourteau d'arachide & $17,10 \pm 0,19^{\mathrm{c}}$ & $32,17 \pm 0,59^{\mathrm{e}}$ & 36,50 & 428,62 \\
Balles de riz & $11,70 \pm 0,22^{\mathrm{d}}$ & $5,51 \pm 0,42^{\mathrm{g}}$ & 61,18 & 372,05 \\
Aliment ponte & $11,94 \pm 0,23^{\mathrm{d}}$ & $14,92 \pm 0,54^{\mathrm{f}}$ & 54,84 & 386,47 \\
\hline
\end{tabular}

Les moyennes situées dans la même colonne et portant les lettres différentes en exposant sont significativement différentes au seuil $P<0,05$. 


\section{DISCUSSION}

Les teneurs en eau des tourteaux de soja analysés sont supérieures aux $10 \%$, trouvées par Debruyne (2001) dans le tourteau de soja aux Etats Unis d'Amérique. La teneur en eau des balles de riz $(12,05 \mathrm{~g} / 100 \mathrm{~g}$ d'aliment), est supérieure à $5,45 \%$ trouvée par Ngom (2004) dans les balles de riz du Sénégal. Les teneurs en eau élevées des aliments seraient dues à l'absorption d'eau par les aliments au cours de l'entreposage, car un environnement qui a une humidité relative élevée (53 à 97\%) et une température ambiante allant de 20 à $30{ }^{\circ} \mathrm{C}$ favoriseraient le transfert d'eau de l'enceinte vers l'aliment (Abdellah, 2004). En outre, cette teneur en eau des aliments serait également due au type d'emballage utilisé pour conditionner le produit, car les aliments sont contenus dans les emballages en polyéthylène qui ne sont pas étanches à la vapeur d'eau ambiante d'où, l'absorption d'eau par le produit. Parallèlement une teneur en eau variant de 8,86 à $16,65 \mathrm{~g} / 100 \mathrm{~g}$ d'aliment indique une forte teneur en matière sèche qui est synonyme d'une teneur élevée en principes nutritifs à savoir : les protéines, les lipides, les glucides et les sels minéraux.

Les cendres représentent l'ensemble des minéraux contenus dans l'échantillon. Les teneurs en cendres des tourteaux de soja B $(5,25 \mathrm{~g} / 100 \mathrm{~g}$ d'aliment), tourteau de soja $\mathrm{C}$ (5,02 g/100g d'aliment) sont comparables aux $5 \%$ trouvé par Debruyne (2001) dans le tourteau de soja aux USA. De plus les teneurs en cendres des farines de poisson des échantillons (farine de poisson E, 18,98 $\mathrm{g} / 100 \mathrm{~g}$; farine de poisson $\mathrm{D}, 16,46 \mathrm{~g} / 100 \mathrm{~g}$ sont comparables à la valeur de $17,17 \%$ trouvée par Bastianelli et al. (2009) dans les farines de poisson en France. Ceci peut s'expliquer par le fait que l'espèce de poisson utilisée pour fabriquer la farine de poisson a une teneur élevée en cendres. La teneur en cendres du tourteau d'arachide $(4,38 \mathrm{~g} / 100 \mathrm{~g})$ est proche de la valeur $5 \%$ trouvée par Batal et al. (2005) et celle des balles de riz $(9,57$ $\mathrm{g} / 100 \mathrm{~g}$ d'aliment) est inférieure à la teneur en cendres de 19,9\% trouvée par Ngom (2004). Les farines de poisson présentent une teneur en cendres élevée de près de $18 \%$ par rapport aux autres aliments analysés. Cette forte teneur en cendres traduit une teneur élevée en sels minéraux, or les sels minéraux sont les éléments indispensables au bon fonctionnement de l'organisme et à la croissance des animaux.

Les teneurs en lipides des tourteaux de soja, sont largement supérieures à valeur de $1 \%$, qui est celle reportée par Debruyne (2001) dans le tourteau de soja aux Etats Unis d'Amérique. Les balles de riz ont une teneur en lipides de $11,7 \mathrm{~g} / 100 \mathrm{~g}$ d'aliment, valeur supérieure à $1,84 \mathrm{~g} / 100 \mathrm{~g}$ des balles de riz analysées par Ngom (2004). Les valeurs des teneurs en lipides des farines de poisson (farine de poisson D, 9,59\%; farine de poisson E, 11,99\%) sont comparables à la valeur de 10,9\% trouvée par Bastianelli et al. (2009) dans les farines de poisson. Ces fortes teneurs en lipides dans le tourteau de soja B et le tourteau de soja $\mathrm{C}$ peuvent s'expliquer par le fait que l'extraction d'huile des graines n'a pas été optimale lors de la presse. Cependant, les lipides sont un principe nutritif riche en énergie car un gramme de lipide apporte environ $9 \mathrm{kcal}$ d'énergie. Les lipides jouent un rôle important dans l'alimentation car ils augmentent la palatabilité par rétention de la flaveur de l'aliment.

Les teneurs en protéines sont inférieures à la valeur de $47 \%$ trouvé par Markal (2011) dans les tourteaux de soja. Ces faibles teneurs en protéines des tourteaux de soja seraient dues à la variété de soja utilisée. Toutefois, on constate que le tourteau de soja C obtenu au niveau de l'unité de presse du GIC est de $36,12 \mathrm{~g} / 100 \mathrm{~g}$, valeur pas très éloignée des valeurs 39,15 et $39,38 \%$ des tourteaux de soja importés (Tourteau de soja A, Tourteau de soja B). D'autre part, les teneurs en protéines des farines de poisson $\mathrm{D}$ 
et $\mathrm{E}$ sont respectivement de 55,69 et 63,73 $\mathrm{g} / 100 \mathrm{~g}$. Ces teneurs sont certes élevées par rapport aux teneurs de tous les aliments analysés mais, restent inférieures à la valeur $76 \%$ trouvée par Bastianelli et al. (2009) dans les farines de poisson en France. Ces faibles teneurs en protéine de nos farines de poisson seraient due au choix de la partie du poisson utilisée ou à l'espèce de poisson car, la valeur nutritionnelle des farines de poisson varie en fonction de ces paramètres sus-énumérés. Pour ce qui est des balles de riz, la teneur en protéine $(5,51 \mathrm{~g} / 100 \mathrm{~g})$ est supérieure à 3,03 g/100g d'aliment trouvée par Ngom (2004) dans les balles de riz. Cette teneur en protéines légèrement élevée dans les balles de riz peut s'expliquer par le fait qu'une petite quantité de brisures de riz et de son de riz se retrouvent dans ces balles de riz, ce qui contribuerait à augmenter la teneur en protéine. En ce qui concerne le tourteau d'arachide, sa teneur en protéine $(32,17 \mathrm{~g} / 100 \mathrm{~g})$ est inférieure aux valeurs de $47,79 \%$ et $45 \%$ trouvées par Nijimbere (2003) et Nir (2003) dans les tourteaux d'arachide. Cette faible teneur serait due à la variété d'arachide utilisée. L'aliment ponte est cet aliment destiné à la nutrition des poules pondeuses en âge de ponte. Il présente une teneur en protéine de 14,92 g/100g. Cette teneur est inférieure à la valeur de $18,5 \%$ recommandée par la FAO (2012) et à $20 \%$ trouvée par Fasuyi et Okeke (2014) dans les feuilles de Tithonia diversifolia.

L'énorme écart de concentration en glucides dans ces aliments est compréhensible car, par essence, les balles de riz sont un aliment riche en glucides (fibres alimentaires) et la farine de poisson est très sollicitée pour sa teneur en protéine. Cependant, l'aliment ponte présente une teneur en glucides relativement élevée, ce qui est appréciable car les glucides représentent la principale source d'énergie nécessaire au métabolisme et fournissent l'énergie nécessaire au fonctionnement du cerveau et du système nerveux et sont impliqués dans l'anabolisme des protéines. Certains glucides rentrent dans la composition de tissus fondamentaux de l'organisme : les cartilages, les acides nucléiques, le mucus, les substances antigéniques (Sguera, 2008). Puisqu'ils sont digérés rapidement, les glucides libèrent leur énergie de façon relativement plus rapide que les protéines et les lipides. Par ailleurs, un aliment présentant un taux élevé en glucides n'est pas synonyme d'un aliment ayant une forte valeur énergétique car, parmi les glucides, il y a des glucides digestes et d'autres indigestes (non métabolisables) par les monogastriques (volailles et porcs). Les glucides indigestes sont les fibres alimentaires qui sont constitués de celluloses et d'hémicelluloses. Les fibres alimentaires sont les résidus de glucides non digérables et indispensables au bon fonctionnement du transit intestinal, c'est-à-dire facilitent la digestion. Ces fibres sont des polymères végétaux non hydrolysables par les enzymes digestibles de l'animal monogastrique en raison, de la nature des liaisons chimiques entre les chaînes.

Les valeurs énergétiques des farines de poisson sont de $341,23 \mathrm{kcal} / 100 \mathrm{~g}$ pour la farine de poisson local et de $348,58 \mathrm{kcal} / 100 \mathrm{~g}$ pour la farine de poisson E. Nijimbere (2003) a trouvé une valeur de $338,1 \mathrm{kcal} / 100 \mathrm{~g}$ pour la farine de poisson. Quant à l'aliment ponte, sa valeur énergétique est de 386,47 kcal/100g. Cette valeur énergétique de l'aliment ponte destiné à l'alimentation des poules pondeuses en âge de ponte est nettement supérieure à 280 $\mathrm{kcal} / 100 \mathrm{~g}$ tel que recommandé par la FAO (2012). En somme, la valeur énergétique très élevée de nos aliments analysés peut-être expliquée par le fait qu'ils ont une teneur très élevée en lipides. Nous rappelons qu'un gramme de lipides fournit 9 kcal d'énergie contrairement aux protéines et glucides qui n'en fournissent que $4 \mathrm{kcal} / \mathrm{g}$. Cependant, en alimentation animale, on dit d'un aliment qu'il est riche en énergie quand il renferme une 
teneur importante en glucide (surtout en amidon). Ainsi, les aliments énergétiques chez les monogastriques sont les céréales (maïs, sorgho, blé, riz). D'autre part, les régimes alimentaires pour les volailles et porcins sont composés principalement d'un mélange de plusieurs aliments tels que les céréales (maïs, riz, blé), tourteaux de soja, dérivés de produits animaux (farine de poisson, os, coquilles, etc), de matières grasses, de prémélanges de vitamines et de minéraux. Tous ces aliments fournissent l'énergie et les nutriments nécessaires pour la croissance, la reproduction et bien-être des animaux. Selon Sauvant (2005), plusieurs formes d'énergies sont retenues des aliments au cours de leurs métabolismes dans l'organisme animale. C'est ainsi qu'il existe, l'énergie brute qui est celle qui est libérée en tant que chaleur, lorsque, la substance est entièrement oxydée.

\section{Conclusion}

Il ressort de cette étude que : le tourteau de soja $\mathrm{C}$ présente la teneur en eau la plus élevée $(16,65 \mathrm{~g} / 100 \mathrm{~g})$. Les farines de poisson sont riches en cendres $(16,46 \mathrm{~g} / 100 \mathrm{~g}$ pour la farine de poisson $\mathrm{D}$ et $18,98 \mathrm{~g} / 100$ pour la farine de poisson $E$ ). Les tourteaux de soja $B$ et $\mathrm{C}$ sont les plus riches en lipides respectivement $(26,96$ et $24,35 \mathrm{~g} / 100 \mathrm{~g})$. Les tourteaux de soja sont les meilleures sources en protéines végétales $(39,38 ; 39,15$ et 36,12 $\mathrm{g} / 100 \mathrm{~g}$ respectivement pour les tourteaux de soja A, B et C). Les farines de poisson sont les meilleures sources en protéines animales $(63,73$ et $55,39 \mathrm{~g} / 100 \mathrm{~g}$ respectivement pour les farines de poisson $\mathrm{D}$ et $\mathrm{E})$. Les balles de riz sont l'aliment le plus riche en glucide $(61,18 \mathrm{~g} / 100 \mathrm{~g})$. Les tourteaux de soja B et C sont les aliments les plus énergétiques respectivement $(465,54$ et $435,05 \mathrm{kcal} / 100 \mathrm{~g})$. Ces données pourraient orienter les fermiers pour le choix des ingrédients de hautes valeurs nutritionnelles et de moindre coût pour la formulation alimentaire des poules pondeuses et porcs.

\section{CONFLIT D'INTERET}

Nous déclarons qu'il n'y a aucun conflit d'intérêt entre les auteurs de ce manuscrit.

\section{CONTRIBUTIONS DES AUTEURS}

ACT a collecté les échantillons. RP et AG ont effectué les analyses aux laboratoires. $\mathrm{RP}, \mathrm{AG}$ et ACT ont rédigé le projet de manuscrit. EF a supervisé toutes activités menées.

\section{REMERCIEMENTS}

Nous remercions le Laboratoire du Centre de Recherche en Alimentation de Nutrition de l'Institut de recherche Médicale et d'étude des Plantes Médicinales de Yaoundé pour l'analyse des échantillons de cette étude.

\section{REFERENCES}

Abdellah Z. 2004. Détermination des mycotoxines dans les aliments et étude de la réduction des aflatoxines par les bactéries lactiques isolées des ferments panaires traditionnels. Thèse de dotctorat, Faculté des sciences Dhar El Mahrez, Université Sidi Mohammed Ben Abdellah, $220 \mathrm{p}$.

AOAC. 1980. (Association of Official Chemists). Official and Tentative Methods of Analysis of the AOAC. AOAC: Washington; 978p.

Batal A, Dale N, Café M. 2005. Nutrient composition of peanut meal. J. Appl. Poult. Res., 14(2): 254-257.

Bastianelli D, Epaku OR, Bonnal L, Grimaud P. 2009. Qualité des matières premières : résultats d'une étude en Afrique de l'Est. Perspectives pour la gestion de la variabilité des matières premières. Revue Africaine de Santé et de Productions Animales, 7: 33-40.

Debruyne I. 2001. Soja: transformation et aspects industriels. Techniques de l'ingénieur, F6030, 12p. 
Donfack P, Seiny-Boukar L, M'biadoun M. 1996. Les grandes caractéristiques du milieu physique. In Agriculture des Savanes $d u$ Nord Cameroun vers un Développement Solidaire des Savanes d'Afrique Centrale, pp. 7-20.

DSCE (Document de stratégie pour la croissance et l'emploi). 2009. Cadre de référence de l'action gouvernementale pour la période de 2010-2020, 167p.

FAO. 2004. Situation mondiale de l'alimentation et de l'agriculture. Collection $\mathrm{N}^{\circ} 35$, Rome, 51p

FAO. 2005. Food and Agricultural Organization of United Nations. FAO Statisticaldatabases. http://faostat.fao.org/

Fasuyi AO, Okeke P. 2014. Extrapolating nutritional potentials of ensiled wild sunflower (Tithonia diversifolia) leaf meal: proximate composition and functional properties. Int. J. Biol. Chem. Sci., 8(1): 8-16.

Jeune Afrique. 2010. Plus d'un milliard de personnes sous alimentées dans le monde. www.jeuneafrique.com/actu. Consulté le le $13 / 07 / 2012$

Markal PA. 2011. Protéines de soja texturées biologique. Fiche technique. www.markal.fr

Ndébi G, Kamajou J, Ongla J. 2009. Analyse des contraintes au développement de la production porcine au Cameroun. Tropicultura, 27(2): 70-76.
Nijimbere A. 2003. Variabilité de la composition chimique et de la valeur alimentaire des matières premières et aliment utilisés et potentiellement utilisables en aviculture dans la zone des Niayes au SénégaI. Mémoire d'ingénieur, ENSA Thiès, 67 p.

Nir. 2003. Cours international sur la production avicole intensive. Alimentation et nutrition des volailles, $124 \mathrm{p}$.

Ngom S. 2004. Ebauche d'un référentiel sur la composition chimique et valeur nutritive des matières premières utilisables en alimentation de la volaille au Sénégal. Thèse de Chimie et Biochimie des produits naturels, 143p.

Sauvant D. 2005. Principes généraux de l'alimentation animale. Institut national agronomique paris-grignon, 159p.

Sguera S. 2008. Spirulina plantensis et ses constituants interêts nutritionnels et activités thérapeutiques. Thèse université Henri Poincare - Nancy1, 197p.

Sonaiya EB, Swan SEJ. 2004. Production en Aviculture Familiale - Un Manuel Technique de FAO, Production et Santé Animales. FAO, Rome, Italy, 134 pages.

SSEPIA/MINEPIA. 2002. Stratégie Sectorielle de l'Elevage, des Pêches et des Industries Animales. Ministère de l'Elevage, des Pêches et des Industries Animales du Cameroun, 94p. 